Meta

Journal des tradlucteurs

Translators' Journal

\title{
Le fichier du traducteur
}

\section{Service de linguistique du Canadien Pacifique}

Volume 15, numéro 2, juin 1970

URI : https://id.erudit.org/iderudit/004091ar

DOI : https://doi.org/10.7202/004091ar

Aller au sommaire du numéro

Éditeur(s)

Les Presses de l'Université de Montréal

ISSN

0026-0452 (imprimé)

1492-1421 (numérique)

Découvrir la revue

Citer cet article

Service de linguistique du Canadien Pacifique (1970). Le fichier du traducteur. Meta, 15(2), 112-115. https://doi.org/10.7202/004091ar d'utilisation que vous pouvez consulter en ligne.

https://apropos.erudit.org/fr/usagers/politique-dutilisation/ 
au XIX ${ }^{e}$ siècle. Mais $s^{\prime}$ il fallait changer d'appellation à chaque mutation que subit un concept, la langue serait constamment à refaire. L'instinct populaire est beaucoup plus sain, lui qui n'hésite pas à continuer d'appeler ferret le bout du lacet même si les fabricants ont substitué depuis longtemps le plastique au métal.

ROBERT DUBUC

\section{LE FICHIER DU TRADUCTEUR}

\section{Bills - Contrats de transport}

Si l'on consulte les dictionnaires de traduction et qu'on vérifie dans Webster, on constate que le bill of lading est une lettre de voiture (transport terrestre) ou un connaissement (transport maritime), et le waybill, une lettre de voiture, une feuille de route, un bordereau ou bulletin d'expédition.

Cela ne vous semble pas très clair? Vous avez parfaitement raison. Poursuivons nos recherches. Le Grand Larousse nous dit que la lettre de voiture est de plus en plus remplacée par le récépissé, document de nature fiscale; d'après le Lamy Transport ${ }^{1}$, la déclaration d'expédition tient lieu de lettre de voiture pour la S.N.C.F. Pour en savoir davantage (ou pour nous y perdre encore plus), nous cherchons dans l'index de Lamy Transport à «bulletin d'expédition»: on nous renvoie à «déclaration d'expédition». Quelle est donc la différence entre lettre de voiture, déclaration d'expédition et bulletin d'expédition ?... Il semble n'y en avoir aucune, plusieurs termes désignant la même réalité. N'allons pas voir les différents usages que font de ces termes les entreprises du Québec; la confusion n'en serait que plus grande.

La traduction de air waybill par lettre de transport aérien suggère une solution possible à ce problème. Pourquoi ne pas parler tout simplement de lettre de transport maritime, lettre de transport aérien, lettre de transport ferroviaire, lettre de transport routier, etc. À l'heure de l'uniformisation des normes de transport à travers le monde, il faudrait aussi songer à la normalisation du lexique.

\section{Corporate}

Cet adjectif, dérivé de corporation, permet de qualifier tout ce qui concerne la société, ou mieux le groupe, dans son ensemble. Ceci établi, les équivalents ne manquent pas. Le premier de ceux-ci est sans doute social qui, selon Robert, qualifie tout élément « relatif à une association de personnes ayant un but commun, des intérêts communs $»$. On aura donc, sur le modèle de raison sociale (corporate name), les comptes sociaux (corporate accounts), et le cachet social ou sceau de la société (corporate seal). Lorsqu'il est évident que l'expression étudiée désigne une action ou un état qui concerne l'ensemble du groupe, on peut se dispenser de traduire corporate. Par exemple, l'expression corporate communications program - il s'agit ici de donner de la société ou du groupe une image susceptible de susciter la demande - peut se rendre par programme d'identification commerciale. De même, l'expression corporate departments, qui désigne les services du siège social par opposition à ceux des diverses succursales, peut se rendre tout simplement par grands services. Il convient ici de rappeler les traductions proposées par

1. L. Brunat, Lamy Transport, tome I, Paris, Services Lamy, 1969, p. 153. 
Radio-Canada pour corporate services (services généraux) et corporate directive (directive générale).

\section{Make bulk/break bulk — Groupage/dégroupage}

Le groupage est une technique de transport permettant à plusieurs expéditeurs de grouper leurs envois de détail (less than carload freight) qui ont la même destination, afin de bénéficier d'un tarif réduit. Inversement, un seul expéditeur peut faire acheminer dans un seul wagon (ou un seul camion) des envois ayant des destinations différentes vers un centre de dégroupage. On peut aussi imaginer le cas d'expéditeurs multiples $(A, B, C)$ qui groupent leurs marchandises pour les envoyer à des destinataires différents $(D, E, F)$. Cette situation donne le schéma suivant:

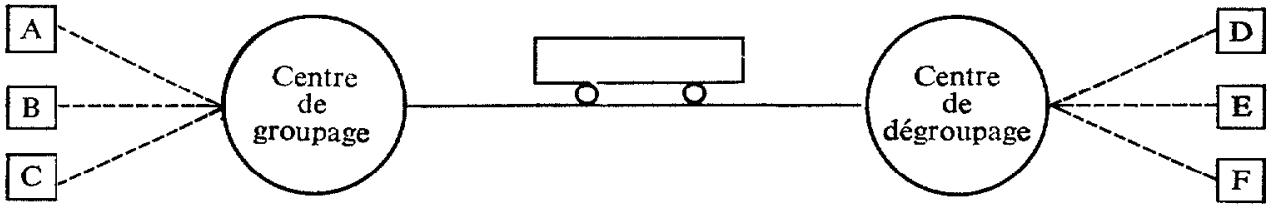

Les expéditeurs $\mathrm{A}, \mathrm{B}$ et $\mathrm{C}$ confient (soit après entente entre eux, soit après des ententes séparées avec un groupeur ou transporteur) leur trafic de groupage (pool car traffic) à un centre de groupage. Les marchandises sont groupées et acheminées par wagons de groupage (pool car) vers un centre de dégroupage proche de leur destination pour être dégroupées et livrées à leurs destinataires respectifs : D, E et $F$.

\section{Pipeline}

La famille des «ducs» semble promise à un avenir aussi brillant que la technique du transport à grande distance par canalisation. Quoique le mot pipe-line ne soit pas plus immotivé en français que micheline, il est intéressant de pouvoir disposer d'autant de termes qu'il existe de variétés particulières d'installations. Aux oléoducs sahariens et aux gazoducs bataves ou soviétiques ont d'abord succédé le pulpeduc (pulp duct) et le granoduc (grain duct), mais voici que la lecture des revues spécialisées révèle l'existence d'un carboduc (coal carrying pipeline) et surtout d'un saumoduc ${ }^{2}$ (salt carrying pipeline).

\section{Rapid transit system - Réseau express régional (Stadtschnellbahn)}

Pour les urbanistes et les spécialistes du transport en commun, il s'agit d'un réseau ferré urbain à grand gabarit (par opposition au métropolitain classique, dit à petit gabarit) qui, tout en irriguant le centre des agglomérations, comporte des antennes en grande banlieue. Ce maxi-métro dont l'exploitation ne diffère pas de celle d'un chemin de fer, a reçu l'appellation officielle française de Réseau express régional, vite abrégée en R.E.R. Cette formule de desserte urbaine à laquelle les pays industrialisés semblent vouloir se rallier (B.A.R.T. de San Francisco, R.E.R. de Paris) n'est autre que celle du bon vieux Stadtschnellbahn (S-Bahn) de Berlin ou de Hambourg. Notons que plusieurs villes canadiennes s'intéressent à la question et que si Toronto a déjà son GO Transit (Government of Toronto Transit), Calgary et Edmonton songent à installer des réseaux ferrés du même genre en utilisant, tout au moins en partie, l'infrastructure ferroviaire existante.

2. La Vie du rail, no 1219 . 


\section{Stopover - Étape}

Il n'est, dit-on, pire intraduisible que le mot qu'on ne veut pas traduire... Stopover semble vouloir se ranger dans cette catégorie. Le grand Webster en donne pourtant une définition bien simple (p. 2251): The act of breaking up one's journey (as by train) with the privilege of continuing on a later conveyance of the same carrier. Il s'agit donc d'un banal arrêt en cours de route. Cependant, l'essor du tourisme aérien a relancé le terme en lui donnant une résonance quelque peu exotique et on l'utilise pour désigner la possibilité offerte au voyageur de s'arrêter en chemin pour visiter les villes qui lui paraissent intéressantes. Le mot escale ayant déjà une signification bien précise - l'avion pouvant faire escale sans que les passagers aient la faculté de s'arrêter — nous lui avons préféré étape. L'expression stopover permitted at Rome peut, par exemple, se rendre par : étape autorisée à Rome. Étape a l'avantage de pouvoir désigner un arrêt au cours d'un voyage en train, en autocar, en bateau, en avion ou en astronef, qui sait?

\section{Piggyback — Rail-route}

Voici un mot-problème. En effet, bien qu'il s'agisse d'une réalité aussi vieille que le rail, le transport par fer des remorques routières (Trailers On Flat Cars, T.O.F.C.) n'a pas recours aux mêmes techniques de part et d'autre de l'Atlantique. Il y a donc un certain danger à rapprocher hâtivement certains termes ${ }^{3}$.

Les chemins de fer américains qui ont, dès le début, adopté un très grand gabarit peuvent transporter sans encombre des remorques routières ordinaires sur des wagons plats ordinaires (fig. 1). Cette technique, simple et pratique, connue sous le nom familier de piggyback dans les pays anglophones et sous la pittoresque expression de transporte a caballito dans les pays hispanophones, est la plus largement utilisée en Amérique.

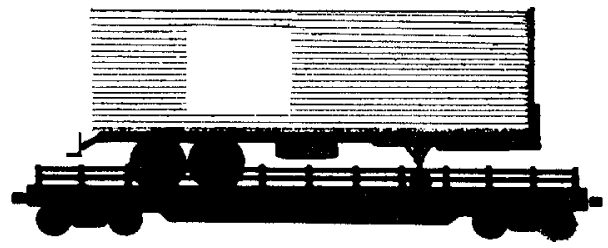

FIG. 1.

En Europe, la question n'est hélas pas si simple... En dépit de l'adoption du 《gabarit passe-partout » $(1=3,15 \mathrm{~m}$ et $\mathrm{h}=4,28 \mathrm{~m})$, il n'est le plus souvent pas possible de charger directement les remorques ordinaires sur les wagons plats ordinaires. Les constructeurs européens ont donc été forcés de concevoir des matériels spécialement adaptés. Pour obvier aux sujétions on a ainsi modifié : a) soit le wagon porteur (technique Kangourou), en installant une sorte de pont-levis qui, en position basse, forme une poche (d'où l'appellation) dans laquelle vient se loger le train arrière de la remorque; $b$ ) soit la remorque (technique dite UFR), en surbaissant celle-ci et en la dotant d'une flèche d'attelage à rabattement automatique; c) soit le wagon porteur et la remorque (technique MC 22) en

3. L'actualité terminologique, vol. 1, no 7, p. 4. 


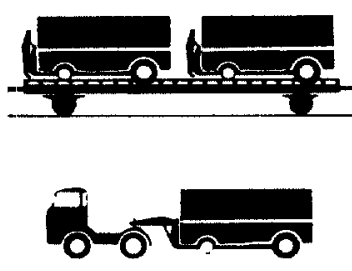

Technique UFR.

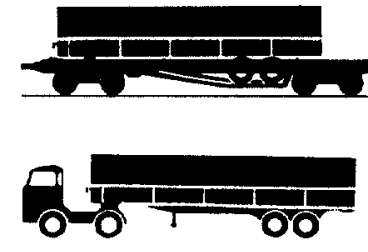

Kangourou

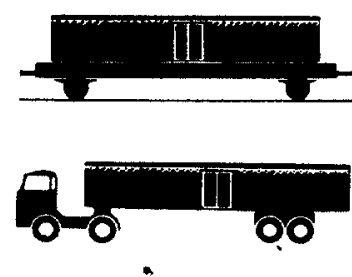

MC 22.

FIG. 2.

équipant celle-ci d'un train arrière amovible (qui reste au chantier de transbordement parfois appelé chantier multitechnique) et en faisant passer la caisse seule sur un wagon spécial très simplifié, analogue aux wagons porte-conteneurs modernes. Cette technique correspond, grosso modo, au Flexi Van américain.

La figure 2, mieux qu'un long discours, résume ces distinctions subtiles. Ajoutons, pour en finir avec la partie technique, que plusieurs administrations ferroviaires européennes s'intéressent à la mise au point d'un wagon plat surbaissé à petites roues que les Chemins de fer fédéraux autrichiens (Ö. B. B.) expérimentent actuellement sur la ligne de l'Arlberg. Comment, dans ces conditions, traduire : piggyback service, piggyback trailer et piggyback ramp?

Une solution satisfaisante au moins s'offre au traducteur. Il peut, en effet, utiliser l'expression rail-route qui, même si certains auteurs ${ }^{4}$ la réservent à la technique UFR, n'en est pas moins générale et particulièrement concrète et commode pour désigner ce type de transport combiné ${ }^{5}$ (intermodal transportation). On parlera donc de transport rail-route, (piggyback service), de remorque rail-route, (piggyback trailer), de chantier rail-route, (piggyback terminal) et de quai ou de rampe rail-route, (piggyback ramp). Dans les cas rarissimes où il importe de distinguer au sein du rail-route la technique particulière utilisée, on pourra, à la rigueur, préciser qu'il s'agit de la technique piggyback en attendant que fleurisse un néologisme de bon aloi dans le genre du ferro-camión en usage au sud du Rio Grande...

Service de linguistique du Canadien Pacifique

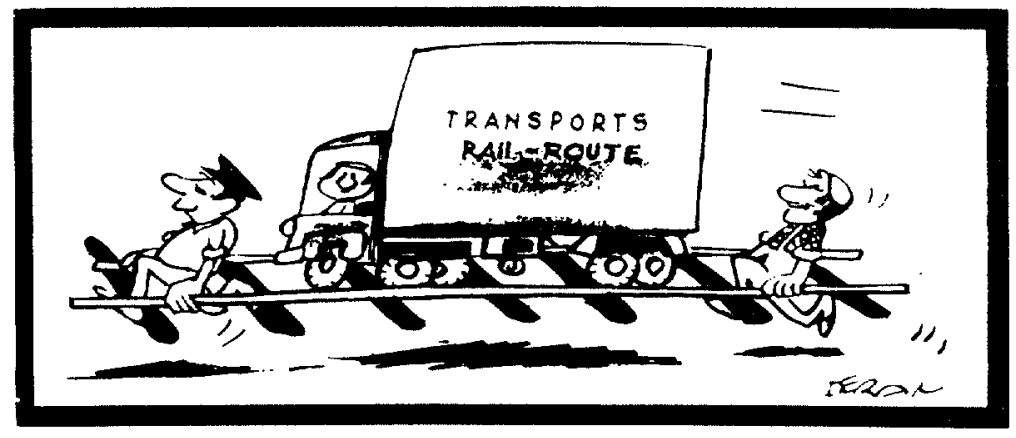

4. Les Chemins de fer, Paris, Larousse, 1964, p. 417.

5. Voir à ce sujet l'article publié dans la Vie du rail, $\mathrm{n}^{\circ} 1231$, p. 7 et suivantes. 